\title{
Intentional penetration of dental implants into the maxillary sinus:
}

\section{a retrospective study}

Accepted: $30 / 12 / 2015$

\section{Omed Ikram Shihab *}

\section{Abstract}

Background and objective: Perforation of the maxillary sinus by a dental implant is an issue of concern by many dental implant clinicians. This study aimed to assess the success rate and consequences of dental implants emerging into the maxillary sinus.

Methods: The maxillary sinus was intentionally perforated for dental implant placement in 35 patients with reduced posterior maxillary bone height ( $4 \mathrm{~mm}$ or more). A total of 70 implants engaged bicortically and emerged into the sinus. Nineteen female and 16 male patients were involved in the study. The patients were followed up for sinus complications and dental implant success.

Results: From the total, only two implants were failed; one during gingival former placement and the other after loading by one year. No patient presented with sinusitis clinically and radiographically. Three patients developed epistaxis just on the day of surgery and became well after on.

Conclusion: Within the limits of this study, dental implant emergence into healthy maxillary sinus is considered a safe and successful procedure.

Keywords: Dental implant; Maxillary sinus; Complications.

\section{Introduction}

The maxillary sinus violation by a dental implant is considered as a potential risk factor for the sinus infection and subsequent implant failure. This violation most probably leads to the occurrence of a postoperative sinus infection. ${ }^{1,2}$ Reduced crestal bone height at the edentulous posterior maxilla results from atrophy of alveolar ridge and pneumatization of the maxillary sinus. ${ }^{3}$ The incidence of the sinus membrane perforation during implant surgeries range from $7 \%$ to $35 \% .{ }^{4,5}$ Sinus membrane perforation is associated with $25 \%$ of all sinus lift and augmentation procedures. ${ }^{6}$ Few researchers investigate the consequences of sinus violation by dental implants. A long-term study, spreading over a period of up to 20 years, indicates that no sinus complication was observed following implant penetration into the maxillary sinus. Furthermore, the absence of occurrence of such complications is related to the maintenance of successful osseointegration. ${ }^{7} \quad A$ histological study found that totally exposed implant was surrounded by respiratory epithelium without inflammatory cells infiltration and the apical parts of the implants became integrated with the bony sinus floor that was mostly lamellar bone. ${ }^{8}$ Perforation of the sinus floor ensures bicortical engagement of the implant with crestal cortical bone and sinus floor cortical bone which is considered the key factor for successful osseointegration. ${ }^{9}$ In the light of many conflicting views, this study aimed to assess the success rate and consequences of dental implants emerging into maxillary sinus intentionally.

Methods
In a five years retrospective study, all
patients with deficient bone in posterior
maxilla were involved. All the patients were
diagnosed by OPG. The maxillary sinus
was intentionally perforated for dental
implant placement in 35 patients with
patients with deficient bone in posterior maxilla were involved. All the patients were diagnosed by OPG. The maxillary sinus implant placement in 35 patients with 
reduced posterior maxillary bone height (4 $\mathrm{mm}$ or less) with sufficient width. This procedure was used in patients who refused sinus lift procedure. A total of 70 implants engaged bicortically and emerged into the sinus. The patients were followed up for sinus complications and dental implant success. All the procedures were explained to the patients, and they gave their consent to the treatment. All the operations were done under local anesthesia using the standard dental implant surgical protocol. During the drilling into the cortex of the maxillary sinus, a good resistant was felt (because of dense cortical bone forming the floor of the sinus). All the implants anchored bicortically with good primary stability (>30 NCm). The implants emerged 1-5 mm into the sinus. Doxicyclin $100 \mathrm{mg}$ Bid used as a standard antibiotic. Mefenamic acid $500 \mathrm{mg} 1 * 3$ used as a pain killer. Dexamethasone $8 \mathrm{mg}$ injection was used on the day of surgery (single injection) for the purpose of reducing the inflammatory reaction in the sinus region. Three different implant systems were used (IDI France, FMD Italy, and Nucleoss Turkey). An OPG was taken for each patient after six months. Then the second stage surgery was done for uncovering the implants. Some patients got one perforation, others more than one, unilaterally or bilaterally. The study was approved by the Scientific and Ethics Committee of the College of Dentistry, Hawler Medical University. Descriptive statistics was used to analyze the data.

\section{Results}

A total of 35 patients, 19 female and 16 male participated in the study. They received 70 implants. The follow-up period ranged from 2-5 years. All patients were evaluated clinically and radiographically (digital OPG). The age ranged from 35-65 years. Three patients developed epistaxis just on the day of surgery and became well after on. Only two implants were failed $(2.86 \%)$. The first failure was in a female patient after loading by one year. It was replaced by another one. The second failure was in a male patient who got bilateral double perforations, two weeks after the second stage surgery. But not replaced because the patient refused to wait another six months. A full arch bridge was made on the rest implants. Other than these two complications no one patient developed any signs and symptoms of sinusitis. OPG showed implants emerging into clear sinus without any pathologic signs. During the period of follow-up, no signs of bone loss or gain were noticed. The emergence into the sinus ranged from 1-5 $\mathrm{mm}$. The height of the implants ranged from $5 \mathrm{~mm}$ to $12 \mathrm{~mm}$. The widths ranged from $3.0 \mathrm{~mm}$ to $5.7 \mathrm{~mm}$. So the size of membrane perforation differed with the size of implants used (Table 1). Unilateral sinus perforation was done in 23 patients. The rest 12 patients had bilateral perforations. Six patients had double perforations and only one patient with triple perforations (Figure 1, 2 and 3).

Table 1: Distribution of dental implants according to site.

\begin{tabular}{lccccc} 
Site & First premolar & 2nd premolar & 1st molar & 2nd molar & 3rd molar \\
\hline Frequency & 7 & 15 & 31 & 16 & 1 \\
Total & & & 70 & & \\
\hline
\end{tabular}




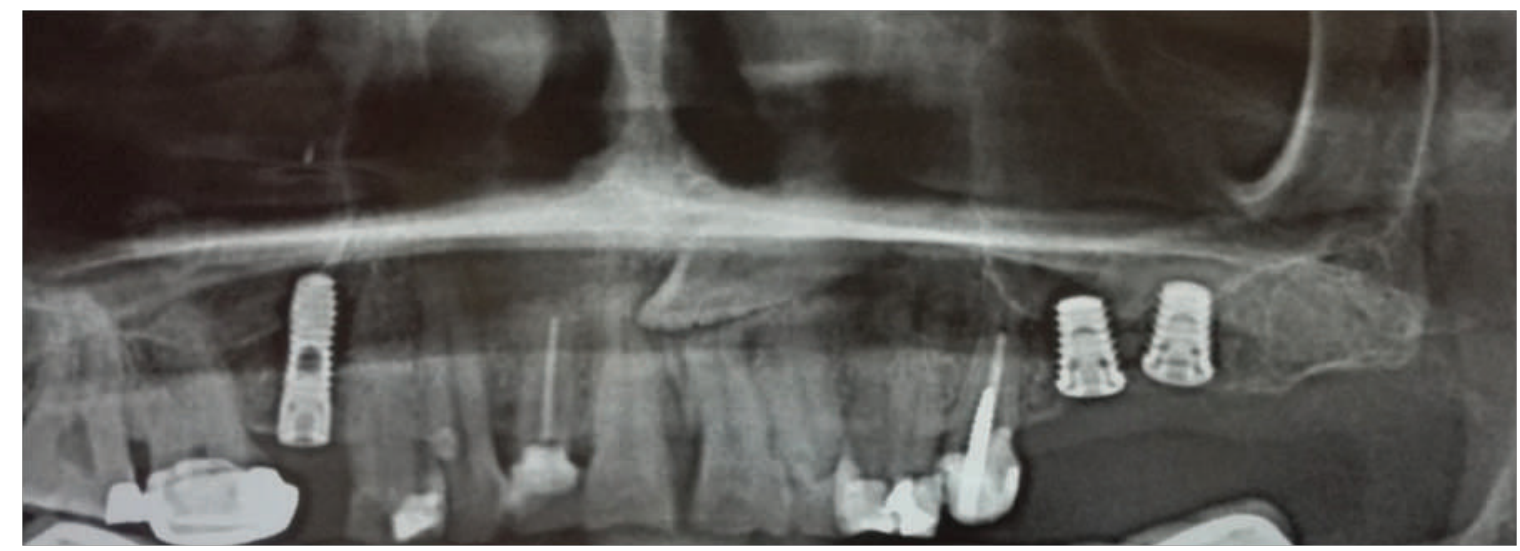

Figure 1: OPG of a case with bilateral perforation. implant length $5 \mathrm{~mm}$. bone height less than $5 \mathrm{~mm}$.

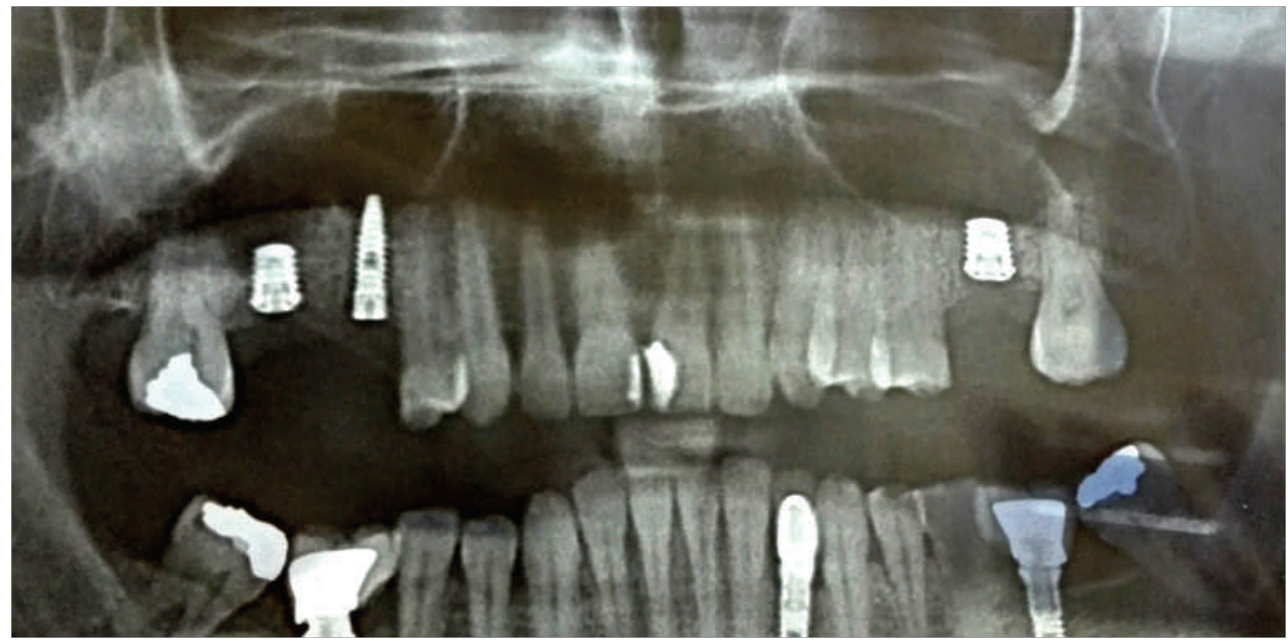

Figure 2: OPG of a case with double perforations on left site and single perforation on the right

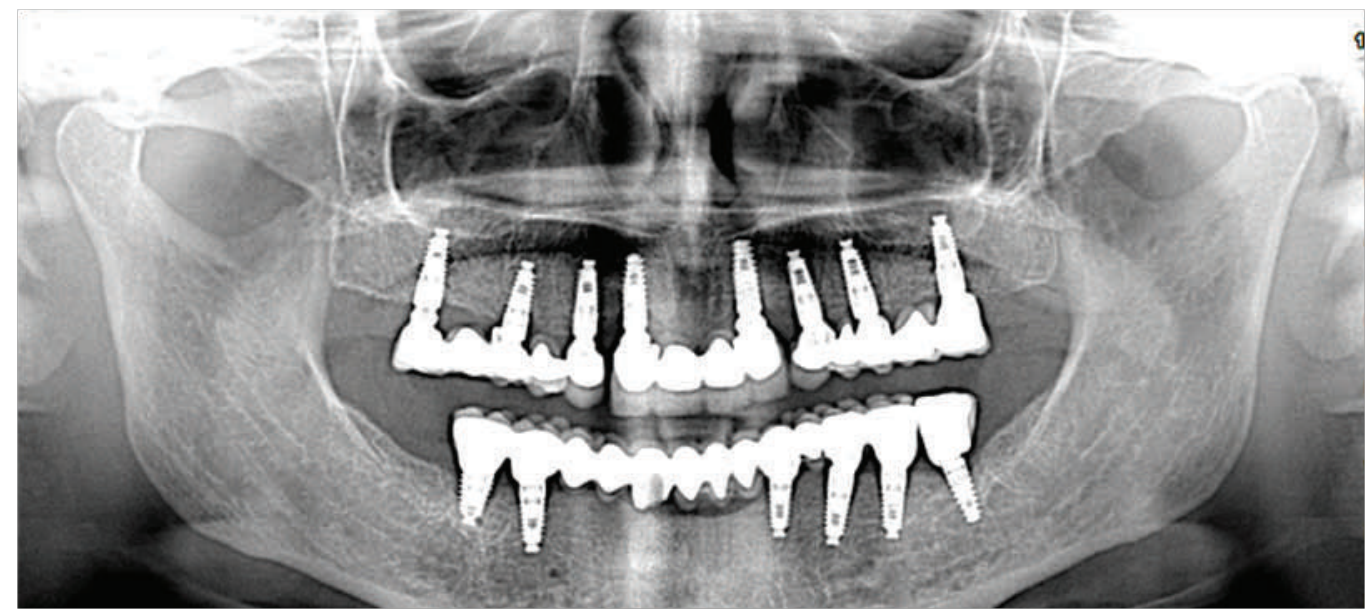

Figure 3: OPG of a case with single perforation on the left side with five years follow-up 


\section{Discussion}

Perforation of the maxillary sinus by the dental implant is an issue of concern by many dental implant clinicians. Many clinicians avoid implant placement in the atrophic posterior maxilla so that they avoid sinus perforation and the possible complications that may occur. It is thought that the implant protrusion into the maxillary sinus can act as a foreign body and thus can cause serious ongoing complications. ${ }^{10}$ In this study, no patient developed any adverse inflammatory reaction in the sinus, and high success rates were achieved. Similar findings were reported by other investigators. ${ }^{7-9}$. Different perforation sizes and depths didn't affect the sinus health, implant osseointegration and success rates. This may be attributed to the fact that Circular epithelium structure similar to gingival cuff formed around the base of the protruding parts and with direct attachment of the membrane to the implants which did not show any sign of inflammation. ${ }^{8}$ No case of implant migration into the sinus was reported. This is may be because bicortical engagement of the implants by the crestal cortex and the cortex of the floor of the sinus granted an excellent primary stability to the implants. ${ }^{9}$ We didn't report any case of maxillary sinusitis. Similar findings were reported by other investigators. ${ }^{7-9}$ In the presence of an acute or chronic maxillary sinusitis, the differential diagnosis must always consider other potential odontogenic and non odontogenic etiologies. ${ }^{7}$ In our study, the implant protrusion into the maxillary was ranged from 1-5 $\mathrm{mm}$, and no difference was reported about complications or success rates. No any previous study reported more than $3 \mathrm{~mm}$ depth of penetration. We believe that sinus lift procedure is more traumatic since there is elevation of a large flap, opening a window in the lateral wall of the sinus, high chance of large membrane perforations which sometimes mandates suturing or use of membranes, and bone graft use. Implant penetration into healthy sinuses before operation, and if necessary postoperative anti-infection management was given, these implants were suggested to remain in place for follow-up observation. ${ }^{11}$ Within the limits of this study, dental implant emergence into healthy maxillary sinus is considered a safe and successful procedure.

\section{Conflicts of interest}

The author reports no conflicts of interest.

\section{References}

1. Cordioli G, Mazzocco C, Schepers E, Brugnolo E, Majzoub Z. Maxillary sinus floor augmentation using bioactive glass granules and autogenous bone with simultaneous implant placement. Clinical and histological findings. Clin Oral Implants Res 2001; 12:270-8.

2. Van den Bergj JP, ten Ruggenkate CM, Disch FJ, Tuinzing DB. Anatomical aspects of sinus floor elevations. Clin Oral Implants Res 2000; 11:256-5.

3. Tatum $H$. Maxillary and sinus implant reconstruction. Dent Clin North Am 1986; 30: 207-29.

4. Chanavaz M. Maxillary sinus: anatomy, physiology, surgery, and bone grafting related to implantology - eleven years of surgical experience (1979-1990). J Oral Implantol 1990; 16:199-209.

5. Shlomi B, Horowitz I, Kahn A, Dobriyan A, Chaushu G. The effect of sinus membrane perforation and repair with Lambone on the outcome of maxillary sinus floor augmentationa radiographic assessment. Int $\mathrm{J}$ Oral Maxillofac Implants 2004; 19:559-62.

6. Barone A, Santini S, Sbordone L, Crespi R, Covani U. A clinical study of the outcomes and complications associated with maxillary sinus augmentation. Int J Oral Maxillofac Implants 2006; 21(1):81-5.

7. Abi Najm S, Malis D, El Hage M, Rahban S, Carrel JP, Bernard JP. Potential adverse events of endosseous dental implants penetrating the maxillary sinus: long-term clinical evaluation. Laryngoscope 2013; 123(12):2958-61.

8. Weijian Z, Binke C, Xin L, Guowu MA. Experimental study on penetration of dental implants into the maxillary sinus in different depths. J Appl Oral Sci 2013; 21(6):560-6.

9. Nooh N. Effect of schneiderian membrane perforation on posterior maxillary implant survival. Journal of International Oral Health 2013; 5(3):28-34.

10. Givol N, Taicher S, Halamish-Shant T, Chaushu G. Risk management aspects of implant dentistry. Int J Oral Maxillofac Implants 2002; 17:258-62.

11. Berengo M, Sivolella S, Majzoub Z, Cordioli G. Endoscopic evaluation of the bone-added osteotome sinus floor elevation procedure. Int $\mathrm{J}$ Oral Maxillofac Surg 2004; 33:189-94. 